\title{
A generic QoS model for web: services design
}

\begin{abstract}
The development, registration, discovery, and invocation of quality Web services are vital for the successful implementation of applications using Web services. Considerable research focuses on quality for Web services. Unfortunately, current research on Quality of Service (QoS) for Web services is concentrated on service users and the implementation stage. This research highlights the importance of incorporating QoS at the design and development stages; the authors propose the introduction of QoS at the same time as functional requirements. However, Web Service Description Language (WSDL) describes the functional elements of a Web service, and QoS is significant for this description. Therefore, the authors propose an extension to the WSDL through a generic QoS metamodel, incorporating QoS specifications into the functionalities. This paper begins by defining the required QoS specifications for the development of quality Web services and explores the potential of the Unified Modeling Language as a technique and notation to specify QoS. To properly integrate QoS in the design, the authors propose extensions to the existing UML QoS profile. The paper concludes with the evaluation of the proposed framework and summarises its advantages.
\end{abstract}

Keyword: QoS metamodel; QoS model; QoS specification; Quality of Service (QoS); UML 